\title{
Patient awareness during critical medical events: communication is key
}

\author{
Renée El-Gabalawy, MA, PhD
}

Received: 28 August 2019/Revised: 16 September 2019/Accepted: 23 September 2019/Published online: 30 September 2019 (C) Canadian Anesthesiologists' Society 2019

The following cases are based on real events with names changed and minor factual alterations to ensure patient privacy

\section{Case 1}

Mary is a middle-aged patient who underwent an orthopedic procedure under spinal anesthesia. Mary's level of consciousness during surgery was not discussed during her preoperative visit with her anesthesiologist, but she expected to remember very little based on her previous experience with spinal anesthesia. Medication for sedation was kept to a minimum in part likely because of the perception that she appeared to be calm and drowsy during the procedure. Afterwards, Mary vividly recalled sounds of the orthopedic hammer and drill and images of her blood on the surgical team. She also remembered not being able to move her legs and that the surgical team discussed topics unrelated to her procedure. Six months later, Mary was diagnosed with post-traumatic stress disorder (PTSD). She was haunted by the vulnerability she experienced during

\footnotetext{
R. El-Gabalawy, MA, PhD ( $\square)$

Department of Anesthesiology, Perioperative and Pain Medicine, Max Rady College of Medicine, Rady Faculty of Health Sciences, University of Manitoba, AE-211 Harry Medovy, 671 William Avenue, Winnipeg, MB R3E 3N4, Canada

e-mail: renee.el-gabalawy@umanitoba.ca
}

Department of Clinical Health Psychology, Max Rady College of Medicine, Rady Faculty of Health Sciences, University of Manitoba, Winnipeg, MB, Canada

Department of Psychology, Faculty of Arts, University of Manitoba, Winnipeg, MB, Canada

Department of Psychiatry, Max Rady College of Medicine, Rady Faculty of Health Sciences, University of Manitoba, Winnipeg, MB, Canada surgery, the feeling of being unimportant, and fearing for her safety.

\section{Case 2}

John is a middle-aged patient who underwent emergency surgery related to gastrointestinal perforation. Afterwards, he went into septic shock and spent several weeks in the intensive care unit (ICU) where he experienced delirium that required sedatives and limb restraints. Following discharge from the hospital, he had difficulty making sense of his experience and had anxiety symptoms related to memories of his delirium-induced delusions and hallucinations. He had trouble differentiating real memories from descriptions of events provided by family members. More than one year after hospital discharge, John was diagnosed with PTSD. He had significant anxiety related to the potential for his mind suddenly "turning on him" again. He had never heard of the term "delirium" prior to treatment for PTSD.

Commentary. As a psychologist specializing in medical trauma, I am referred patients with a range of psychological difficulties related to a medical event perceived as traumatic. These events include acute onset of an adverse health event or critical illness, perioperative complications such as anesthetic awareness, and medical errors, among others. The psychological manifestations related to these traumas vary, and may consist of anxiety, depression, and other stress-related conditions including PTSD. Illness-induced PTSD is a diagnostic classification that has garnered considerable attention recently, with $6.5 \%$ of those with PTSD reporting a life-threatening illness-induced trigger (i.e., the index trauma causing PTSD). ${ }^{1}$ Although psychological symptomatology can vary widely in patients who experience medical trauma, there are commonalities to the experience. One of the most common clinical features is the psychological effect related 
to "provider silence", that is, the failure of the healthcare professional to provide adequate explanation regarding procedural expectations or subsequent adverse experiences. I exemplify this important clinical issue in the above two cases that are relevant to perioperative anesthesia care.

Anesthesiologists generally appreciate the potential psychological implications following unintended awareness during general anesthesia and there is considerable literature on this topic. ${ }^{2}$ What is less well understood in the anesthesia literature are the consequences of consciousness in the context of intravenous sedation (i.e., monitored anesthesia care), and local and/or regional anesthesia. ${ }^{3}$ In these circumstances, it is accepted that the patient's level of consciousness (and recall) may be variable and that awareness may, in fact, be intentional. Nevertheless, there are potentially traumatic consequences of this awareness (or alertness), which require consideration. Preliminary research suggests that a considerable number of patients with such awareness may experience distress (78\%) and negative long-term psychological outcomes (40\%) including PTSD, comparable with those who experienced unintentional awareness during general anesthesia. ${ }^{4}$

In the above case involving Mary, her PTSD was directly linked to the unexpected level of awareness she experienced along with the images and sounds she was exposed to which precipitated her perception of threat. Perceived degree of threat is an important predictor of postoperative PTSD. ${ }^{5}$ In the other case, John experienced delirium, a known predictor of PTSD $^{5}$ that comprises a number of distinct symptoms that can include disturbing and fragmented "flashback" memories of their delusional state. Importantly, John was not debriefed about the hallucinations and delusions he experienced in the ICU nor how they were associated with his critical illnessneither at hospital discharge or during follow-up appointments. Over time, his post-traumatic stress symptoms were exacerbated by the pervasive fear that his mind was failing him.

Had Mary been appropriately informed prior to surgery about the level of awareness she could experience under spinal anesthesia, including the sensation of being unable to move her legs while still hearing and seeing what was going on around her, it is possible that she would have been spared of PTSD (or that her symptoms would not have been as severe). Similarly, if John had received information about his experience of delirium and had been provided with appropriate resources, he too might have been spared of PTSD. Empirically supported PTSD theories emphasize the role of maladaptive processing of traumatic events relating to fragmented autobiographical memory, subjective sense of current threat, and (of particular relevance to these cases) a lack of contextual information. ${ }^{6}$ Taking the time to communicate clearly to patients regarding anticipated or possible perioperative experiences, and providing information following perioperative complications, may mitigate the risks of poor psychological outcomes. ${ }^{4}$ In support, preoperative educational initiatives including reviewing and clarifying expectations and providing information about the procedure and anticipated experiences have been shown to significantly reduce perioperative anxiety. ${ }^{7}$ In addition, studies emphasize the importance of providing timely information to patients (and their families) who experienced delirium to reduce the associated psychological distress that may occur afterwards. ${ }^{8}$

Almost every patient I have treated who has experienced emotional trauma related to their medical experience has lamented over the insufficient and inadequate communication and information they received from their healthcare providers. This is typically an important component of their symptom presentation and is frequently a theme that is integrated into their maladaptive cognitions and mood, a core symptom of PTSD. This lack of communication and information can manifest as pervasive mistrust of healthcare professionals that can extend to other areas of their life (e.g., mistrust of authority) and perpetuate avoidance behaviors that further negatively impact their healthcare (e.g., not attending follow-up appointments). Several emerging interventions exist for psychological effects of medical trauma such as PTSD that might be beneficial particularly if delivered early by mental health professionals, ${ }^{9}$ but the role of the anesthesiologist in prevention of postoperative psychological symptomatology may be critical. The unintentional psychological impact of provider silence can be profound and a few minutes of timely, clear communication may completely alter a patient's mental health trajectory.Conflicts of interest None declared.

Editorial responsibility This submission was handled by Dr. Steven Backman, Associate Editor, Canadian Journal of Anesthesia.

\section{References}

1. El-Gabalawy R, Mota N, Sommer JL, Edmondson D. Prevalence of illness-induced posttraumatic stress disorder in the United States. Psychosom Med 2018; 80: 783-5.

2. Tasbihgou SR, Vogels MF, Absalom AR. Accidental awareness during general anaesthesia - a narrative review. Anaesthesia 2018; 73: $112-22$.

3. Mashour GA, Avidan MS. Intraoperative awareness: controversies and non-controversies. Br J Anaesth 2015; 115: i20-6.

4. Kent CD, Mashour GA, Metzger NA, Posner KL, Domino KB. Psychological impact of unexpected explicit recall of events occurring during surgery performed under sedation, regional 
anaesthesia, and general anaesthesia: data from the Anesthesia Awareness Registry. Br J Anaesth 2013; 110: 381-7.

5. El-Gabalawy R, Sommer JL, Pietrzak R, et al. Post-traumatic stress in the postoperative period: Current status and future directions. Can J Anesth 2019; . https://doi.org/10.1007/s12630-019-01418-4.

6. Brewin CR, Holmes EA. Psychological theories of posttraumatic stress disorder. Clin Psychol Rev 2003; 23: 339-76.

7. Wilson CJ, Mitchelson AJ, Tzeng TH, et al. Caring for the surgically anxious patient: a review of the interventions and a guide to optimizing surgical outcomes. Am J Surg 2016; 212: 1519.
8. Partridge JS, Martin FC, Harari D, Dhesi JK. The delirium experience: What are the effects on patients, relatives and staff and what can be done to modify this? Int J Geriatr Psychiatry 2013; 28: 804-12.

9. Birk JL, Sumner JA, Haerizadeh M, et al. Early interventions to prevent posttraumatic stress disorder symptoms in survivors of life-threatening medical events: a systematic review. J Anxiety Disord 2019; 64: 24-39.

Publisher's Note Springer Nature remains neutral with regard to jurisdictional claims in published maps and institutional affiliations. 